\title{
From Responsible Gambling to Responsible Innovation?
}

\author{
Richard Woolley
}

This non-peer reviewed entry is published as part of the Critical Gambling Studies Blog. Visit an interactive version of this blog at: https://criticalgamblingstudies.blogspot.com/2021/02/fromresponsible-gambling-to.html

The gambling industry is knowledge, research, technology and innovation driven. It uses knowledge from multiple scientific fields to design products that are attractive to consumers. It both absorbs state of the art scientific and technological research and conducts its own in-house research and development programmes. It uses research data to segment customers to better target marketing and rewards and to experiment with new games and gambler experiences. The ways it uses research generates a mix of positive and negative social, economic, and health impacts.

The gambling industry can, from this perspective, be viewed as a powerful knowledge actor with a highly privileged position and capacity to obtain and use data and information, both to monitor performance and innovate to shape the future. Gamblers using online accounts or land-based loyalty programmes, or other forms of membership style access, provide a treasure trove of data to their chosen gambling providers. Client data from businesses delivering gambling to customers (B2C) maps out the preferences and behaviours of customer segments allowing customisation of the gambling experience. When shared with game designer and equipment supplier partners (B2B) this data facilitates the updating of consumer products and experiences according to 'real world' information.

While the gambling industry in many countries has enjoyed a period of largesse in relation to the permitted expansion of legal gambling markets in recent decades, the process of product innovation in gambling remains relatively closely monitored and tightly regulated by governments worldwide. This leads to one of the major complaints of the gambling industry: why is our particular form of 'addiction by design' treated differently than that promoted by Netflix or by Facebook, or any other consumer product that seeks to maximise customer engagement? This pleading points toward one of the major reasons why commercial gambling remains tightly controlled, despite its size, financial power and political influence. The gambling industry, in the main, has never shed its adversarial approach to regulation or its resentment toward societal criticism stemming from the incontrovertible harm that commercial gambling does produce.

This blogpost does not go back over the ground of who is responsible for harm production among gamblers. Rather it asks the question whether the gambling industry could do better, and should do better, in reducing harm production in the interests of its clients and ultimately of itself. I will suggest that the gambling industry, as a powerful knowledge actor, could do more and that it is absolutely in its best interests to do so. The gambling industry often looks to be in rude health from the outside, but the value of B2C gambling businesses has been slowly trending downwards in many markets, whereas 
B2B gambling businesses have continued to be seen as attractive by investors. The increasing importance of environmental, social and governance (ESG) criteria and principles for responsible investment (PRI) are changing the way industries and commercial sectors are assessed and rewarded by financial markets and institutional investors. In such a context, the old model of squeezing every last drop out of vulnerable clients before they are discarded has to be consigned to history - and in some enlightened companies probably it already is - in favour of an understanding of the client base as an asset that has to be sustained, nurtured and protected.

From this broader perspective, it can be argued that the $\mathrm{B} 2 \mathrm{C}$ strata of the gambling industry has not become as ethically proactive in relation to problem gambling among its customers as it needs to. The B2B strata - particularly companies that persist with the old model of fast, dissociative game experiences, rather than slowing games down and making them more entertaining - can be argued to have not become sufficiently responsible in its innovation processes and practices. However, the overall industry reluctance to recognise player safety and product safety as two sides of the same coin may be a strategy that is reaching the end of its usefulness. Just like industries and companies increasingly at risk due to the fossil fuel pollution they produce, the gambling industry may be reaching a reckoning with its own 'kick the can down the road' struggle to frame gambling harm as simply a function of poor or impaired decision-making on the part of some of its clients.

The sense that the gambling industry might be missing its opportunity to get ahead of market and regulatory curves has been exacerbated by recent decisions taken by the regulator in the UK. Having been described by the National Audit Office as struggling to keep pace with technological advance in the industry, the UK Gambling Commission surprised many by announcing a raft of changes to online

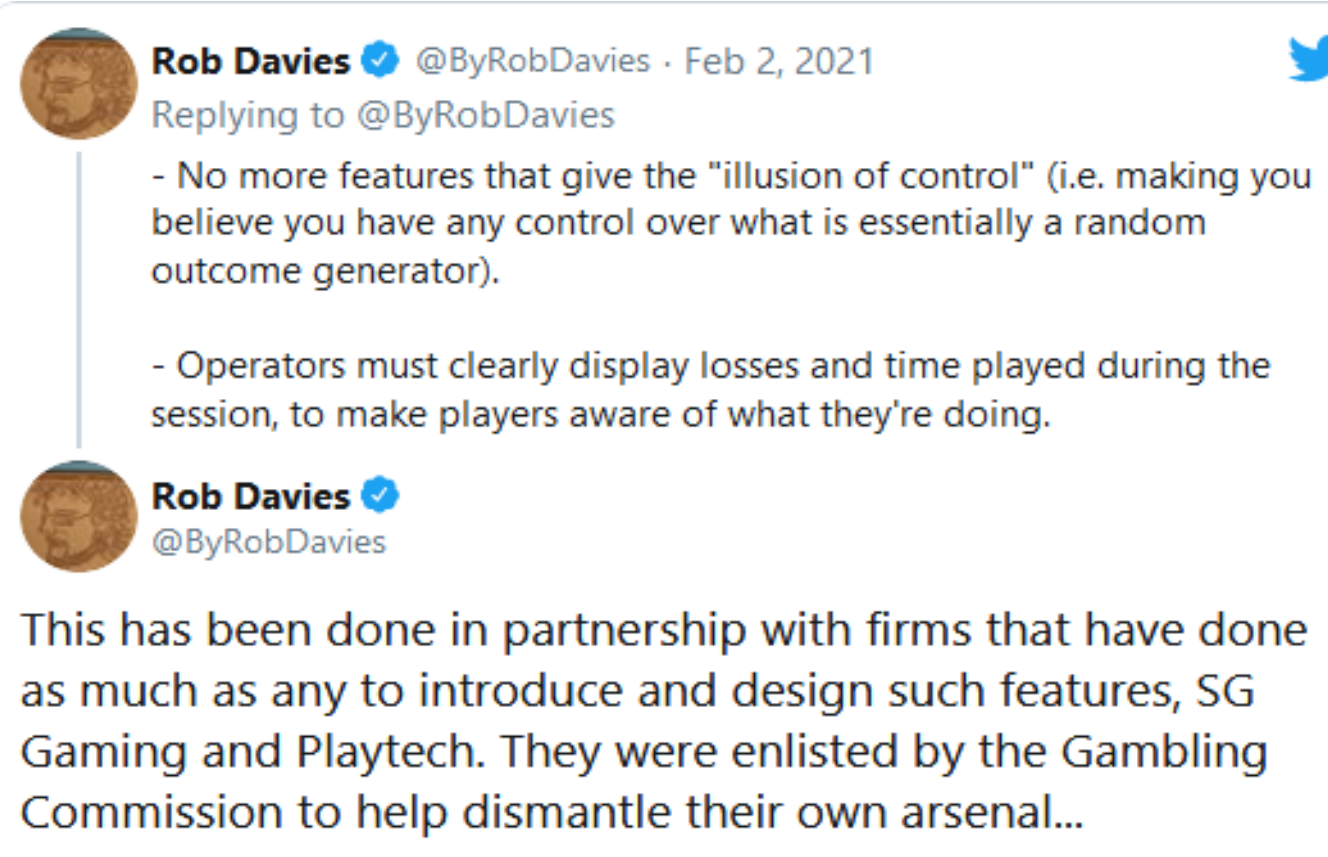

8:30 AM · Feb 2, 2021

$\bigcirc \quad 3 \& 1 \&$ Copy link to Tweet 


\section{Critical Gambling Studies Blog}

gambling that must be implemented in nine months (by October 31, 2021). Crucially, the changes detailed have the objective of making online gambling games safer by design.

The main changes to the Remote gambling and software technical standards (RTS) requiring action include:

- the elimination of auto-play from online slots games;

- removal of options to play multiple slots games simultaneously;

- a minimum game-cycle of 2.5 seconds (same as Category C land-based fixed odds betting terminals (FOBTs);

- elimination of celebrations/positive rewards for net stake loss bets (losses disguised as wins (LDWs); and

- the elimination of features (such as reel-stopping) that give the illusion of control over game outcomes.

From a public health perspective such changes are to be welcomed, but can hardly be said to make online slots games safe for consumers. From an industry perspective such changes will not be welcomed as they will impact on game design significantly (particularly eliminating LDWs) and reduce revenue at least in the short term. In other words, nobody is entirely satisfied and the stand-off continues.

So what is the way forward? One way forward would be for the gambling industry to embrace its own responsibility, as the most powerful knowledge actor with unrivalled insights into possible innovation pathways, to transition toward valuing product safety. Why wait to cooperate on product safety until being required to by regulators? Surely approaching product safety as integral to game design and development would reduce the risk of imposed regulatory changes that are more negative for the industry than they need to be in order to satisfy consumer protection objectives?

Adopting a responsible innovation (RI) approach would require the industry to adopt more open and transparent processes of innovation, that take into account more complex sets of values than simply the building of a better mousetrap. The key to RI lies in sustained and consistent processes of engagement with interested stakeholders and concerned groups to address social and ethical concerns along the innovation pathway. In the case of the gambling industry, where the adverse consequences of gambling can have a ripple effect felt across many other sectors of society, responsibility driven engagement around future innovation pathways would ideally include gamblers, governments, regulatory authorities, industry competitors and collaborators, and the health and financial sectors, at a minimum.

$\mathrm{RI}$ recognises that power and agency accrue to those who control knowledge and shape how it is used. By opening up deliberations about innovation RI encourages reflection and anticipation around potential negative impacts, holding dominant actors to collective account. Whereas negative consequences of R\&l have been typically discreetly sidelined in categories such as 'unforeseen consequences' or 'negative externalities', RI demands an active effort involving the full stakeholder community in order to avoid or redress negative impacts.

So, returning to the question at the top of this post, what is the next frontier for gambling technology, and what are the chances that it can be governed through a responsible approach to innovation? 


\section{Critical Gambling Studies Blog}

Could the gambling industry be convinced by ESG in markets and regulatory risk to drop its usagainst-the-world approach to controversies such as problem gambling?

As the designers of games and powerful information actors, the gambling industry is essential to any reform of existing products or responsible approach to future design processes. Companies in the industry are uniquely positioned to best understand the relationship between their client gamblers and their gambling product offer. For online slots, or any gambling game played by means of an access card, gambling data collected is always for both player and product, gambler and game. Betby-bet, deposit-by-deposit, game choice-by-game choice data builds intimate and detailed gambler profiles, average session metrics, and maps the amounts and sources of every gamblers' funds. Time spent on device, bet sizes, game volatility, bonus choices, and more compile rigorous product performance profiles. But the crucial point is that these player and product data are entirely interdependent, they are part of each other. These data are what enable companies to construct a comprehensive 'diagram of interactivity' between their client base and their products. This diagram of interactivity enables industry to know a lot about what makes their business tick - but also what makes some of its clients sick.

Adopting a responsible innovation approach in the gambling industry would require the integrity of the diagram of interactivity to be maintained in all discussions of problem gambling and consideration of interventions. Responsibility falls short if it is only applied to persons, but never to the products that are integral to their behaviour. Let's take online slots gambling as an example. Customer A's account data at their favourite online casino shows they are spending an increasing amount of time gambling on the site. The frequency and length of their slots gambling sessions have increased. Customer B can be observed to be depositing increasing amounts of money at the same casino. They have recently added new credit card deposit sources to their account and the losses incurred in their slots gambling sessions have increased. Both customers could be considered ready for referral to 'responsible gambling' services due to their changed behaviour and the emergence of potential for (or actual) harms.

Maintaining the full diagram of interactivity available to industry would mean not severing the available information about the behaviour of these customers from the configuration of the gambling products that both made possible and co-produced their behaviour. Rather it would be used to inform us better; for example that Customer A has migrated from hopping around among different types of casino games and a range of slots to an almost exclusive focus on locking into low volatility slots games, betting multiple lines (multiple bets) that generate frequent small rewards and reinforcements (including through losses disguised as wins). The design of these low volatility slots are the perfect companion allowing Customer A to spend longer periods gambling without radically changing their pattern of expenditure. Pursued over a longer time, however, such a strategy carries an emerging risk of a dissociative effect that can itself become the raison d'etre for gambling activity - in which case we might also expect that gradual increases in monetary losses will start to occur. Customer B on the other hand might be revealed to have moved from regularly sampling a range of slots to gambling almost exclusively on high volatility games. Excessive expenditure could be the outcome of pursuing the adrenaline rush experience of sizeable wins, of upping stakes in anticipation after periods of receiving low or no rewards, potentially ending up in a situation of chasing losses that have exceeded intended spend. Adding new sources of funds to their account at the same time that their high volatility slots gambling is increasing should raise a red flag. 
Many parts of the gambling industry are already in possession of comprehensive information on their clients' gambling budgets, their product preferences and their gambling consumption. These data are already being used to train artificial intelligence (Al) applications and to design algorithms that can enhance the gambling experience for consumers. What such Al tools can, and should, also be doing is integrating into customer account management systems and gambling product designs to systematically identify emerging indications of unsafe gambling outcomes. The gambling industry could initiate an ongoing and iterative innovation process involving these technologies with the objective of improving player and product safety and reducing harm production. Expectations would need to be managed and, from an RI perspective, these processes would have to be open and the designs of algorithms explained, debated, and contributed to by regulators, by expert-throughexperience gamblers, by consumer protection authorities, academic experts, and other relevant stakeholders.

The gambling industry has the data, technological infrastructure, and human intellectual capacity to engage in better and more responsible innovation processes. It has the awareness of looming market and regulatory risks if it does not elect to do so. But does it, in the end, have the political will to overcome its historically adversarial understanding of its relationship with governance and external societal forces? Does it have the self-awareness to realise that unrestrained pursuit of the most direct path possible to revenue growth and private profit may not be the best model to ensure the sustainability of the industry in the coming decades? Can it see the writing on the wall for marketing via sport, including through sponsorship of the world's highest profile football leagues? Can it imagine itself embracing broader sets of public values as many of the largest and most successful companies and industries are in the process of doing? Can enlightened actors, whether researchers, CEOs, or companies, move from the periphery to put a responsible innovation agenda onto the big boardroom tables for discussion and debate? Or will one sector of the industry, such as internet/remote gambling, take the lead and change its own (innovation) path?

An industry's model of innovation is only locked-in until someone or something disrupts it. As the strength of disruptive market, technology, regulatory, and social forces builds, those parts of the gambling industry which take their future into their own hands by adopting a responsible approach to innovation may be best placed to control how that future looks.

Richard Woolley is a sociologist working at INGENIO (CSIC-UPV) Universitat Politècnica de València in Spain. Most of his current work focuses on the relationships between science, innovation and society. He has a long standing interest in the sociology of gambling, particularly the machine gambling industry. 